\title{
Isopodes Aselloïdes stygobies d'Espagne récoltés par J. Notenboom et I. Meijers, III - Le genre Proasellus: A - Espèces oculées ou microphtalmes*
}

\author{
Jean-Paul Henry et Guy Magniez \\ Biologie Animale et Générale, Université de Bourgogne, 6,Bd. Gabriel, 21000 Dijon, France
}

Keywords: Asellidae, Proasellus, microphthalmous species, origin of stygobionts, Spain

\begin{abstract}
Samples collected in underground waters of Spain by Ine Meijers and Jos Notenboom demonstrated the presence of some oculated asellids previously known: Proasellus coiffaiti, $P$. coxalis, and $P$. meridianus, and also four new stygophilous species, more or less depigmented, with different stages of regression of the ocular system: $P$. beticus n. sp., $P$. ortizi $\mathrm{n}$. sp., $P$. aragonensis $\mathrm{n}$. sp., and $\boldsymbol{P}$. ebrensis $\mathrm{n}$. sp. These forms represent relict populations of ancient epigean species. The anophthalmous species of the Aquitanian Basin, Pyrenees, and Iberian Peninsula are more closely related to $P$. beticus and $P$. ortizi than to the Recent epigean species $P$. coxalis and $P$. meridianus.
\end{abstract}

\section{Résumé}

Le matériel récolté par Ine Meijers et J. Notenboom montre la présence dans les eaux souterraines d'Espagne d'Asellides oculés déjà connus: Proasellus coiffaiti, $\boldsymbol{P}$. coxalis et $\boldsymbol{P}$. meridianus, mais également d'espèces stygophiles non décrites, plus ou moins dépigmentées et à appareil oculaire en voie de régression: $\boldsymbol{P}$. beticus n. sp., $\boldsymbol{P}$. ortizi n. sp., $\boldsymbol{P}$. aragonensis n. sp. et $\boldsymbol{P}$. ebrensis n. sp. Ces formes doivent être considérées comme des populations résiduelles d'anciennes espèces de surface. Les espèces stygobies du bassin aquitain, des Pyrénées et de la péninsule ibérique sont beaucoup plus étroitement apparentées à $P$. beticus et $P$. ortizi qu'aux espèces épigées récentes $P$. coxalis et $P$. meridianus.

\section{Généralités}

Dans nos études précédentes (Henry \& Magniez, 1987, 1988), sur les Asellides d'Espagne, nous avons successivement envisagé les formes des genres Synasellus et Bragasellus. Tous deux sont strictement endémiques de la péninsule ibérique. De plus, le premier est uniquement composé d'espèces stygobies, dépigmentées et anophtalmes, tandis que le second ne comporte plus que 2 espèces oculées lusitaniennes connues, alors que toutes les autres sont anophtalmes et souterraines.

Le présent travail est consacré au genre Proasellus Dudich, 1925, dont la situation est très différente de celle des genres précédents:

- Un très grand nombre d'espèces sont déjà connues (Henry et al., 1986),

- L'aire géographique du genre est immense: de la Grande-Bretagne à l'Atlas marocain en latitude, de la péninsule ibérique et de Madère à l'Asie Mineure et au Caucase en longitude.

- I comprend des espèces pigmentées-oculées largement répandues dans les eaux de surface: $P$. coxalis (Dollfus, 1892) et $P$. meridianus (Racovitza, 1919), par exemple, mais aussi de nombreuses espèces anophtalmes et dépigmentées. Certaines, uniquement troglobies, sont endémiques et cantonnées dans un massif karstique déterminé (Proasellus spelaeus [Racovitza, 1922] du massif des Arbailles, Pyrénées-Atlantiques, par exemple); d'autres, plutôt phréatobies, ont diffusé dans les nappes alluviales d'un réseau hydrographique; les dernières enfin, à la fois troglobies et phréatobies, peuvent avoir une vaste aire de répartition, car elles

\footnotetext{
* Groundwater Crustaceans of Spain, 14.
} 
sont capables de se répandre activement dans les nappes alluviales des bassins hydrographiques contigus par l'intermédiaire des aquifères karstiques ( $P$. cavaticus [Leydig, 1871], par exemple, qui s'est étendu dans le bassin du Rhône, ceux du Rhin et de la Meuse et jusqu'au Sud de la Grande-Bretagne).

Certaines formes du genre occupent une position intermédiaire: microphtalmes et plus ou moins largement dépigmentées (Ermin, 1944); elles se localisent souvent dans les écotones entre eaux épigées et hypogées, niches semi-obscures: sources, résurgences ou pertes, puits, etc. (cas de $P$. racovitzai Henry \& Magniez, 1972b, des exsurgences du Goueil-di-Her, Haute-Garonne, France). Elles représentent souvent des relictes d'une espèce autrefois largement répandue dans les eaux superficielles de la région considérée, mais qui a été refoulée vers l'amont du réseau hydrographique, soit par l'installation plus récente d'une nouvelle espèce épigée plus expansive dans le bassin, soit par l'assèchement progressif du climat, qui fait disparaître saisonnièrement les eaux de surface permanentes, soit encore par le réchauffement postglaciaire de ces eaux dans les régions de basse altitude. Isolées dans ces biotopes-limites et dans un état biologique métastable, ces populations sont vraisemblablement destinées, soit à péricliter avec le temps, soit à évoluer vers un statut de stygobie en donnant une ou plusieurs nouvelles espèces anophtalmes et dépigmentées.

Dans la péninsule ibérique, les Proasellus étaient essentiellement connus par des travaux de limnologie, mettant en évidence l'espèce cosmopolite $P$. coxalis et ses différentes sous-espèces ou par des travaux de biospéologie citant des espèces troglobies du Portugal central (travaux de Braga, 1946, 1956, puis de Afonso, 1982), de la région pyrénéocantabre (travaux de Henry \& Magniez, 1972a, b), puis des espèces phréatobies du S.E. de la péninsule (Henry \& Magniez, 1982) ou du N.E. (Sabater \& De Manuel, 1988).

Dans un but de clarté, nous étudierons d'abord les formes présentant, soit des yeux fonctionnels, soit des résidus oculaires visibles, réservant l'étude des formes parfaitement anophtalmes, phréatobies ou troglobies, à une publication ultérieure. Naturellement, cette division répond à une simple commo- dité, car chez les formes microphtalmes, la partie dioptrique des ommatidies peut être totalement atrophiée, si bien que ces formes doivent être considérées comme physiologiquement aveugles. Aux échantillons capturés par J. Notenboom et I. Meijers en 1983, 1984 et 1985 et par J. Notenboom en 1986 (Notenboom \& Meijers, 1985a,b; Notenboom, 1986), nous avons adjoint ceux récoltés en Andalousie, durant l'année 1985, par P. van den Hurk et R. Leys (Van den Hurk \& Leys, 1986). Nous les remercions très vivement de nous avoir confié ce matériel.

\section{Partie taxonomique}

Proasellus coxalis (Dollfus, 1892)

[= Proasellus banyulensis (Racovitza, 1919)]

Espèce très polytypique, normalement pigmentée et oculée (Fig. 38), vivant dans tous les types d'eaux superficielles, parfois même oligohalines. Après Racovitza (1919), qui détermina comme Asellus banyulensis des Asellides épigés de la province d'Alicante, Margalef (1950) le cite de Majorque, puis en 1953, des provinces de Gerona, Barcelona, Castellon, Alicante et caractérise ainsi ses biotopes: "... En aguas estancadas o circulantes, comun especialmente entre hojas muertas de caducifolios pudriendose en el agua, de las que parecen alimentarse ...".

Son aire s'étend sur tout le pourtour de la Méditerranée. Les localités-types se trouvent près du lac de Tibériade. De nombreuses îles: Madère, Majorque, Hyères, Corse, Sardaigne, Sicile, îles grecques, possèdent des populations particulières, si bien qu'une multitude de sous-espèces ont été décrites. Un peu partout, des populations obscuricoles s'isolent dans des sources, puits et rivières souterraines. On peut même rencontrer des populations souterraines en état d'instabilité génétique, avec des individus dépigmentés, les uns encore munis d'yeux, les autres anophtalmes (puits du Beausset, dans le Var, France, par exemple), mais l'architecture des pléopodes copulateurs montre que ces isolats appartiennent toujours à cette espèce 
(Henry, 1967). On est donc fondé à affirmer que les espèces stygobies d'Europe et d'Afrique du Nord ne dérivent pas de $\boldsymbol{P}$. coxalis, mais d'espèces de Proasellus oculés plus anciennes.

Localités et matériel. - Numéro de collection pour toutes ces stations: Zoölogisch Museum Amsterdam (ZMA) Isop. 105.517.

Prov. Alicante:

Station 84/2-25 = 84/3-10 (07/02 et 04/03/1984): puits au N. de la route Benidoleig-Orba, $0,7 \mathrm{~km}$ de Benidoleig, $18 \mathrm{~km}$ N.O. Calpe, alt. $105 \mathrm{~m}$, temp. 16,5 et $15,0^{\circ} \mathrm{C}$. Quelques individus oculés. Les pléopodes 1 et 2 sont typiques.

Station 84/2-26 = 84/3-11 (07/02 et 04/03/1984): puits à 50 $\mathrm{m} \mathrm{S}$. du précédent. Identiques aux individus de station 84/2-25.

Station 84/2-27 (07/02/1984): puits à 0,4 km S. du Río Girena, Orba, $20 \mathrm{~km}$ N.O. Calpe, alt. $85 \mathrm{~m}$, temp. $15,8^{\circ} \mathrm{C}$. Semblables aux précédents.

Station 84/2-29 (07/02/1984): puits à $50 \mathrm{~m} \mathrm{~S}$. du Río Girena, Sanet y Negrals, $20 \mathrm{~km}$ N.O. Calpe, alt. $80 \mathrm{~m}$, temp. $16,6^{\circ} \mathrm{C}$. Identiques aux précédents.

Station 84/2-30 (07/02/1984): puits au N. de la route DeniaVergel, 1,5 km de Denia, $22 \mathrm{~km}$ N.N.E. Calpe, alt. 5-10 m, temp. $17,8^{\circ} \mathrm{C}$. Individus petits, oculés et légèrement pigmentés, pléopodes 1 et 2 typiques.

Station 84/3-12 (04/03/1984): résurgence temporaire, Cueva del Agua, versant N. de la Sierra del Montgo, Denia, $20 \mathrm{~km} \mathrm{~N}$. Calpe, alt. $320 \mathrm{~m}$, temp. $12,6^{\circ} \mathrm{C}$. Semblables aux précédents.

Station 84/2-8 (02/02/1984): puits La Hoya 2, Torrevieja, 44 $\mathrm{km}$ S. Alicante, alt. $15 \mathrm{~m}$, temp. $18,0^{\circ} \mathrm{C}$.

Prov. Murcia:

Station 84/2-5 (01/02/1984): puits au $\mathrm{S}$. de la route, Casas Blancas, $26 \mathrm{~km}$ E.S.E. Murcia, alt. $100 \mathrm{~m}$, temp. $18,8^{\circ} \mathrm{C}$.

Prov. Málaga:

Station 84/7-24 (26/07/1984): puits près du pont de la route Benaoján-Ronda, sur le Río Guadalevin, $65 \mathrm{~km}$ O. Málaga, alt. $300 \mathrm{~m}$, temp. $19,8^{\circ} \mathrm{C}$. Presque totalement dépigmentés. Yeux à 3 ocelles quasi incolores. Pléopodes 1 et 2 typiques.

Station A85/7-31 (12/07/1985): puits à Colmenar, $22 \mathrm{~km}$ N.N.E. Málaga, alt. $800 \mathrm{~m}$, temp. $18,8^{\circ} \mathrm{C}$. Individus oculés.

Station A85/7-19 (08/07/1985): puits à El Velerin, Estepona, $70 \mathrm{~km}$ S.O. Málaga, alt. $5 \mathrm{~m}$, temp. $19,8^{\circ} \mathrm{C}$. Petite taille, yeux bien pigmentés, mais corps très clair.

Station A85/7-21 (08/07/1985): puits à Punta Pinillos, 70 km S.O. Málaga, alt. $20 \mathrm{~m}$, temp. $19,0^{\circ} \mathrm{C}$. Individus oculés.

Prov. Huelva:

Station A85/6-1 (22/06/1985): puits à La Plancha, Cartaya, $17 \mathrm{~km} \mathrm{O}$. Huelva, alt. $18 \mathrm{~m}$, temp. $18,3^{\circ} \mathrm{C}$. Yeux bien pigmentés, mais coloration du corps presque nulle.

Remarques. - Les stations hypogées de $P$. coxalis répertoriées (Fig. 41) sont toutes proches du littoral marin, depuis la province d'Alicante jusqu'à celle de Huelva et à des altitudes généralement faibles, avec des eaux de température relativement élevée (jusqu'à $19,8^{\circ} \mathrm{C}$ ) et de chlorinité souvent impor-

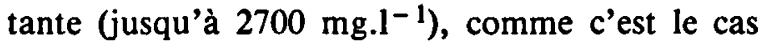
pour les populations de $P$. coxalis épigés des basses plaines alluviales. Ceci montre qu'il s'agit de peuplements plus récents que ceux des autres Proasellus de la péninsule ibérique.

Les individus de ces populations de puits et résurgences ressemblent souvent beaucoup aux $P$. coxalis africanus (Monod, 1924) que l'on trouve dans de nombreux puits au Maghreb. On peut se demander si l'Homme n'a pas participé activement à la dispersion de $P$. coxalis sur le pourtour de la Méditerranée, durant l'Antiquité et la colonisation arabe, en embarquant dans les navires des réserves d'eau douce prélevées dans les sources, résurgences et ruisseaux, près du littoral marin, au cours de ses périples; la présence de l'espèce dans pratiquement toutes les grandes îles méditerranéennes exige que la question soit posée, comme elle l'a été pour expliquer sa présence à Madère.

\section{Proasellus meridianus (Racovitza, 1919)}

(Figs. 32, 39)

Habituellement pigmenté et oculé (Fig. 39), cet Aselle est l'espèce normale des eaux épigées (sources, ruisseaux, mares, étangs de l'ouest de la France, parmi les algues, les mousses, les roseaux, etc.; Bretagne, Charentes, bassin aquitain, dont la Garonne et ses affluents pyrénéens: Henry \& Magniez, 1983). Racovitza (1919) cite sa présence à Sare, près de la frontière d'Espagne. Plus au Nord et à l'Est (Pays-Bas, Grande-Bretagne, N.E. de la France) il est plus localisé, souvent refoulé vers les sources et exsurgences par l'envahisseur d'origine asiatique Asellus aquaticus (Linnaeus, 1758), plus prolifique et moins exigeant sur la qualité des eaux. On remarquera son absence du bassin méditerranéen, sauf exception (Gouffre de l'Oeil Doux à St.-Pierre-sur-Mer, Aude, Fig. 41).

$P$. meridianus est très obscuricole et donne de nombreuses populations hypogées: puits de Bretagne, rivières souterraines (Padirac et SaintGéry), aqueducs souterrains, avec parfois des individus dépigmentés et microphtalmes. Il s'enfonce 
profondément dans l'hyporhéique à grands interstices des rivières (galets de la Dordogne, par exemple). L'espèce n'était pas connue en Espagne. Au Portugal, l'espèce $P$. xavieri de l'étang de Fermentelos, près de Coimbra, décrite par Braga en 1956 semble correspondre à une population isolée de $P$. meridianus. Les prospections récentes montrent que l'espèce est présente dans les eaux souterraines, entre la frontière française et la région de Santander et qu'elle pénètre dans le haut bassin de l'Ebre (station 84/4-5), mais il conviendrait d'effectuer des prospections dans les eaux épigées pour connaître l'extension exacte de l'espèce dans les zones atlantiques de l'Espagne.

Localités et matériel. - Numéro de collection pour toutes ces stations: ZMA Isop. 105.519.

Station 83/8-2 (11/08/1983): source captée au N. de La Busta, Alfoz de Lloredo, $33 \mathrm{~km}$ O.S.O. Santander, alt. $100 \mathrm{~m}$. Un mâle adulte de $5,1 \mathrm{~mm}$; teinte brun-clair, yeux noirs normalement développés.

Station 84/4-5 (07/04/1984): puits à Palazuelos de Cuesta Urría, Merindad de Cuesta Urria, $60 \mathrm{~km}$ N.N.E. Burgos, alt. 600 $\mathrm{m}$, temp. $8,4^{\circ} \mathrm{C}$. Environ 60 individus de pigmentation variable (du jaunâtre au beige ou au brun-clair), yeux normaux. La taille des mâles atteint $7 \mathrm{~mm}$, celle des femelles est inférieure à $5 \mathrm{~mm}$; 2 femelles ovigères de $4 \mathrm{~mm}$ (13 oeufs) et $3,2 \mathrm{~mm}$ (10 oeufs), 2 femelles à marsupium vide de 3,6 et $3,1 \mathrm{~mm}$.

Station 84/6-29 (17/06/1984): Sondage Bou-Rouch dans le Río Bidasoa au pont de Legasa, Bértiz-Arana, $34 \mathrm{~km} \mathrm{~N}$. Pamplona, Prov. Navarra, alt. $120 \mathrm{~m}$, temp. $15,9^{\circ} \mathrm{C}$. Individus beige-brunâtres avec yeux normaux: 4 mâles de 5,$8 ; 4 ; 3,9$ et 3,8 $\mathrm{mm} ; 2$ femelles de 3,3 et 3,1 mm; 2 immatures de 2,2 et $1,9 \mathrm{~mm}$.

Station 84/6-41A = 83/12-2 (20/06/1984 et 01/12/1983): exsurgence pérenne et rivière souterraine de la Cueva Argatxa, Gautéguiz de Arteaga, sur la Ria de Guernica, 24 km N.O. Bilbao, Prov. Vizcaya, alt. $10 \mathrm{~m}$, temp. 13,2 et $13,9^{\circ} \mathrm{C}$. Lot de 52 individus fortement dépigmentés, y compris au niveau du céphalon, simplement beige-clair; yeux noirs normaux. Mâles atteignant $5,6 \mathrm{~mm}$ et femelles $4,5 \mathrm{~mm}$. Une femelle ovigère de $3,6 \mathrm{~mm}$ avec 12 oeufs et deux femelles à marsupium vide de 3,3 et 3,1 $\mathrm{mm}$.

Station 84/8-13 (26/08/1984): Puits au S. du barrio San Juan, Castañeda, $18 \mathrm{~km} \mathrm{S.O.} \mathrm{Santander,} \mathrm{alt.} 40 \mathrm{~m}$, temp. $18,1^{\circ} \mathrm{C}$. Un mâle immature de $2,8 \mathrm{~mm}$, une femelle de $3 \mathrm{~mm}$ et un juvénile de $1,2 \mathrm{~mm}$, normalement pigmentés et oculés.

\section{Proasellus coiffaiti Henry \& Magniez, 1972}

(Figs. 33, 40)

Cet Aselle a été récolté en 1946 par le regretté Dr. Henri Coiffait, dans le ruisseau de la grotte de
Sare (Pyrénées-Atlantiques). Légèrement pigmenté (brun-clair), les yeux noirs sont bien développés. La taille maximale des 5 individus capturés atteignait $8,1 \mathrm{~mm}$. On pouvait penser qu'il s'agissait d'une espèce endémique du bassin de la Nivelle. Or, en 1983 , notre collègue $B$. Lebreton en a capturé des spécimens dans au moins quatre résurgences de la région de Chalosse (entre Peyrehorade et Aire-surAdour, bassin de l'Adour, Fig. 41). Certains étaient beaucoup plus grands (mâle de $14 \mathrm{~mm}$ ) et plus pigmentés que ceux de la localité-type, ce qui nous a permis de dire que $P$. coiffaiti est la quatrième espèce épigée d'Aselles de France (les autres étant: Asellus aquaticus, Proasellus coxalis et $P$. meridianus).

Localités et matériel. - $\boldsymbol{P}$. coiffaiti a été découvert en Espagne dans une seule station frontalière:

Station 84/6-28 (16/06/1984): petite source dite Fuente Aizpara, sur le flanc N. du mont Aizpara, Zugarramurdi, Prov. Navarra, 48 km N.N.E. Pamplona. La station se trouve à quelques $\mathrm{km}$ seulement à l'est de la station-type des grottes de Sare et également dans le bassin de la Nivelle, alt. $320 \mathrm{~m}$, temp. $12,6^{\circ} \mathrm{C}$. Lot capturé: 58 mâles adultes de 11 à $3,9 \mathrm{~mm}$, taille moyenne de $7,98 \mathrm{~mm} ; 24$ femelles adultes de 7,3 à $5,4 \mathrm{~mm}$ (moyenne de 6,53 mm), comprenant 11 femelles ovigères de 7,3 à $6 \mathrm{~mm}$ (moyenne de $6,5 \mathrm{~mm}$ ), plus 5 femelles à marsupium vide de 6,8 à $5,4 \mathrm{~mm}$ (moyenne de $6,34 \mathrm{~mm}$ ), plus 8 femelles à oostégites de repos sexuel de 7,2 à $6 \mathrm{~mm}$ (moyenne de $6,59 \mathrm{~mm}$ ). Il y a également 5 femelles immatures de 3,8 à $5,8 \mathrm{~mm}$, quelques jeunes de 1-2 mm et des larves marsupiales libérées accidentellement. (No. ZMA Isop. 105.516.)

Remarques. - Les individus sont très semblables à ceux. de Sare et l'espèce est bien reconnaissable à l'aspect du céphalon (Fig. 40), nettement plus pigmenté que le corps sur ses marges antérieure et latérales, ménageant une plage blanche médiane et postérieure, assez semblable à celle de $\boldsymbol{P}$. meridianus, ainsi qu'à la chétotaxie générale très abondante. Les pléopodes 1 et 2 mâles (Fig. 33), également très pileux, ont une architecture très caractéristique. Les yeux sont parfaitement développés, formant un bombement à la surface du céphalon, avec 4-5 ommatidies et doivent être fonctionnels. Corps cristallins bien développés et pigment formant une cupule autour des éléments photorécepteurs.

$P$. coiffaiti conservant toutes les caractéristiques 
d'une espèce épigée, il serait indispensable de connaître son éventuelle répartition dans les eaux superficielles du pays basque.

Proasellus beticus n. sp.

(Figs. 1-8, 34)

Cet Aselle provient de stations obscures relativement proches du littoral méditerranéen, entre les provinces de Castellón et Málaga. Son nom spécifique est lié à sa localisation actuelle dans la région bétique.

Localités et matériel. - Numéro de collection pour les cinq stations: ZMA Isop. 105.515.

Station 84/5-31 (08/05/1984): puits, calle Huerta, Albocácer, $42 \mathrm{~km} \mathrm{~N}$. Castellón, alt. $540 \mathrm{~m}$, temp. $10,7^{\circ} \mathrm{C}$. Station-type. Individus adultes beige, yeux noirs à 4 ommatidies. Quatre mâles de 7,8; 7,6 (holotype); 7,2 et $6,8 \mathrm{~mm}$; une femelle ovigère de 4 $\mathrm{mm}$ avec 20 oeufs (allotype); une femelle de $4,2 \mathrm{~mm}$ avec oostégites réduits et trois jeunes de $1 ; 1$ et $1,6 \mathrm{~mm}$.

Station 84/5-49A (12/05/1984): rivière souterraine: Tunnel dels Sumidors, Vallada, $70 \mathrm{~km} \mathrm{~S}$. Valencia, alt. $460 \mathrm{~m}$, temp. $14,6^{\circ} \mathrm{C}$. Deux mâles adultes de 6,6 et $4,2 \mathrm{~mm}$; une femelle à marsupium vide de $3,7 \mathrm{~mm}$; une femelle de $4 \mathrm{~mm}$ à oostégites réduits et un jeune de $2 \mathrm{~mm}$. Individus jaunâtres avec céphalon plus teinté, 2-3 ommatidies brunes.

Station 84/2-22 (06/02/1984): petite source: Font dels Asmiraors, Benimaurell, Vall de Laguart, $55 \mathrm{~km}$ N.N.E. Alicante, alt. $440 \mathrm{~m}$. Environ 60 mâles, femelles et subadultes dont les plus grands dépassent à peine $3 \mathrm{~mm}$, plus 16 jeunes d'environ 1,1 $\mathrm{mm}$. Teinte beige-clair à blanc-jaunâtre, céphalon plus teinté, yeux à 2 ommatidies, bien pigmentés, même chez les jeunes.

Station 84/2-23 (06/02/1984): captage au S. du Río Girena, Fleix, à quelques $\mathrm{km}$ de $84 / 2-22$ et $55 \mathrm{~km} \mathrm{~N}$.N.E. Alicante, alt. $220 \mathrm{~m}$, temp. $10,0^{\circ} \mathrm{C}$. Cinq mâles de 5,$8 ; 4,9 ; 4 ; 3,6$ et $3,5 \mathrm{~mm}$, plus un juvénile de $2,9 \mathrm{~mm}$. Individus jaunâtres avec 2 ommatidies brunes.

Station 84/7-29 (27/07/1984): résurgence temporaire, Cueva del Agua, Cerro de la Motillas, Cortes de la Frontera, $90 \mathrm{~km}$ O.S.O. Málaga, alt. $300 \mathrm{~m}$, temp. $14,2^{\circ} \mathrm{C}$. Les individus sont presque totalement dépigmentés, les yeux présents et colorés.

Description. - Aspect général: corps trapu et élargi au niveau du péréion, chétotaxie abondante (Fig. 1); pigmentation tergale jaunâtre à beige, plus marquée sur le céphalon (avec des taches blanchâtres alignées sur sa moitié postérieure, Fig. 2) et la base des antennules. Yeux apparemment fonctionnels, avec partie dioptrique comprenant 4 ommatidies et pigmentation oculaire abondante. Coxopodites dé- bordant largement les zones pleurales des tergites.

'Appendices céphaliques: antennules courtes, hampe de 4 et fouet de 7 articles dont les 3 distaux portent une lame olfactive. Antennes avec fouet de plus de 50 articles. Mandibules avec processus inciseur plus développé à gauche, lacinia mobilis à 4 denticules, suivie d'une rangée de 12 tiges (13 à droite); lobe molaire très proéminent avec des formations pectinées en position distale; palpe triarticulé sétigère, normal. Maxillules avec 13 épines distales sur le lobe externe et 5 fortes soies ramifiées sur le lobe interne. Maxilles trilobées normales avec sur chacune une rangée de nombreuses soies falciformes.

Maxillipèdes légèrement pigmentés, rétinacle du protopodite comportant jusqu'à 8 crochets; épipodite partiellement bordé de soies lisses marginales; palpe à 5 articles normalement développés et sétigères.

Péréiopodes I relativement grêles et allongés, avec propodite peu renflé à marge palmaire subrectiligne. Péréiopodes IV du mâle très raccourcis et tordus en crochet nuptial chez le mâle adulte (comme chez $P$. coxalis ou $P$. meridianus). Péréiopodes $V$ à VII de plus en plus longs vers l'arrière avec dactylopodite portant 3-4 épines sternales. Papilles génitales du mâle normales sur le dernier sternite thoracique.

Pléopodes I mâles: protopodite subquadrangulaire, court et portant 1-2 crochets rétinaculaires; exopodite moyennement allongé et garni de soies marginales uniquement courtes et lisses (Fig. 3), comme chez $P$. meridianus.

Pléopodes II mâles: protopodite subquadrangulaire et petit, avec un groupe de soies plumeuses sur la marge interne, formant rétinacle (Fig. 4a); exopodite très long, avec article distal légèrement élargi distalement et portant distalement de très longues soies plumeuses, avec une aire pilifère sur la face interne; endopodite pyriforme allongé; une apophyse tergale très massive et courte, dont l'axe est pratiquement perpendiculaire à celui de l'organe; goulot de diamètre important, orienté en direction externe, légèrement évasé en corolle, avec marge de l'orifice efférent un peu réfléchie (Fig. 4b), ce qui est l'indice d'une parenté certaine avec $P$. meridianus. 

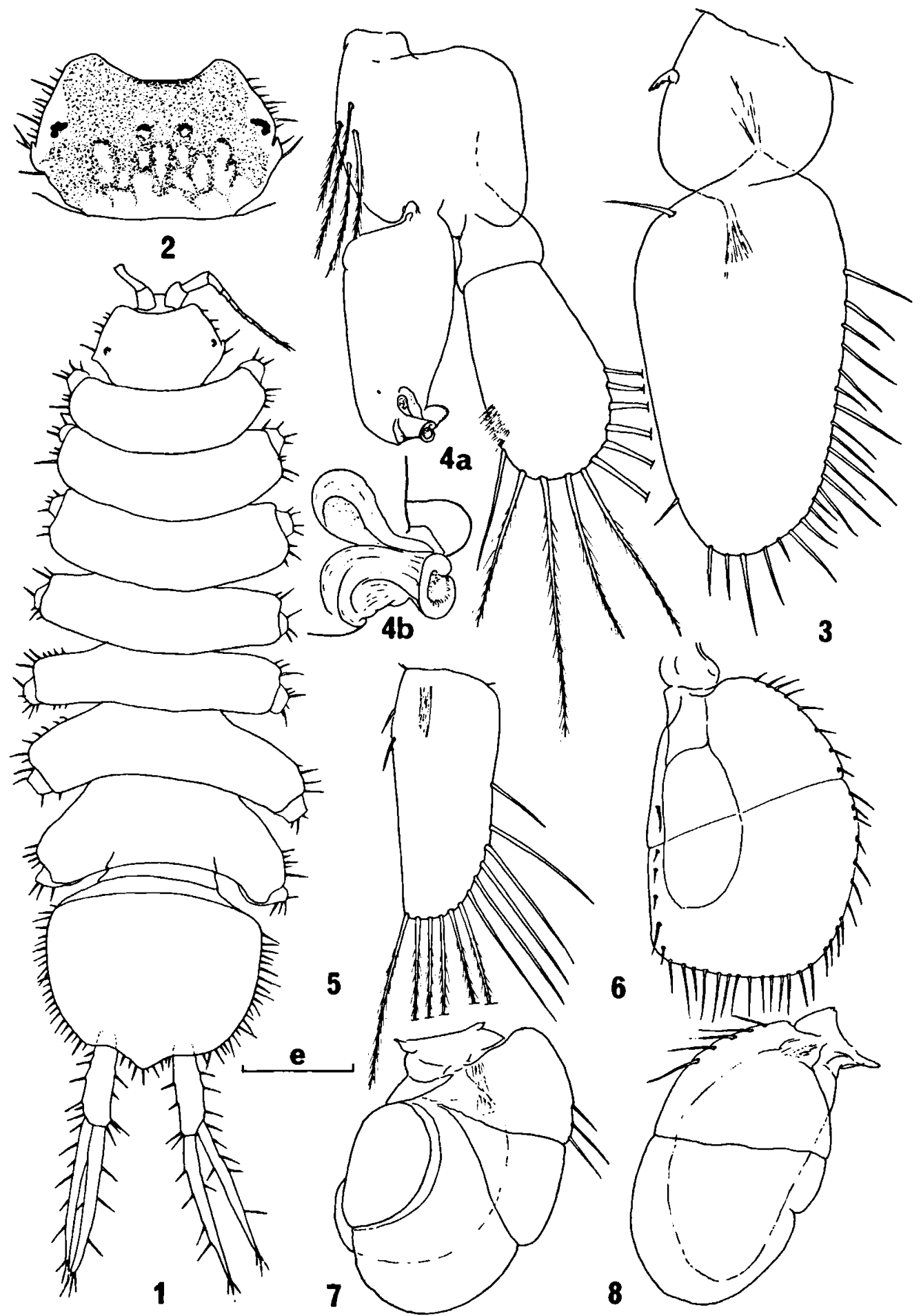

Figs. 1-8. Proasellus beticus n. sp.: 1, habitus d'un mâle de $6,8 \mathrm{~mm}$ env., e $=1 \mathrm{~mm} ; 2$, céphalon du même, e = 0,7 mm; 3 , pléopode I du même, $\mathrm{e}=150 \mu \mathrm{m} ; 4 \mathrm{a}$, pléopode II gauche, face sternale, du même, e $=150 \mu \mathrm{m} ; 4 \mathrm{~b}$, extrémité de l'endopodite du précédent, e $=50 \mu \mathrm{m} ; 5$, pléopode II d'une femelle adulte de $4,2 \mathrm{~mm}, \mathrm{e}=200 \mu \mathrm{m} ; 6$, pléopode III de 1, e $=560 \mu \mathrm{m} ; 7$, pléopode IV du même, $\mathrm{e}=465 \mu \mathrm{m} ; 8$, pléopode $\mathrm{V}$ du même, $\mathrm{e}=465 \mu \mathrm{m}$. 
Pléopodes II femelles: allongés (Fig. 5), indépendants, avec de petites soies rétinaculaires lisses; marge distale-externe très arrondie avec de très longues soies plumeuses.

Pléopodes III: exopodite (opercule) biarticulé avec marge externe et distale totalement bordée de courtes soies, lisses sur la marge externe, mais finement plumeuses sur la marge distale (Fig. 6); endopodite charnu et respiratoire petit et oblong.

Pléopodes IV: exopodite subovalaire; marge externe du premier article pourvue de fines sétules et de 3 soies lisses (Fig. 7): la linea conjungens et la linea transversalis délimitent un triangle plus important que chez $P$. meridianus; les deux parties de la linea areæ forment une lunule bien nette et l'area charnue est aussi développée que chez $P$. meridianus. Endopodite à article distal charnu et respiratoire subcirculaire.

Pléopodes V: exopodite régulièrement ovalaire; marge externe de l'article proximal avec environ 6 soies lisses; linea duplex légèrement courbe; linea articularis peu marquée (Fig. 8); endopodite charnu et respiratoire à peine plus court que son exopodite et régulièrement ovalaire.

Uropodes: nettement plus longs que le pléotelson (Fig. 1), protopodite remarquablement allongé; endopodite dépassant légèrement l'exopodite.

Affinités. - Malgré son aspect assez semblable, $P$. beticus n. sp. ne semble pas avoir une parenté immédiate avec l'espèce $P$. coxalis commune dans la même aire. L'architecture de ses pléopodes la rapproche, d'une part, de toute une série d'espèces dépigmentées et totalement anophtalmes déjà connues de cette même région: $P$. solanasi Henry \& Magniez, 1972, magnifique espèce troglobie de la Cueva del Gato, Málaga, P. jaloniacus Henry \& Magniez, 1978, petite espèce interstitielle du Río Jalón, Alicante, $P$. comasi Henry \& Magniez, 1982, du sous-écoulement du Río Guadalmedina, Málaga, P. escolai Henry \& Magniez, 1982, de l'exsurgence de Deifontes, Granada, $P$. lagari Henry \& Magniez, 1982, du sous-écoulement du Río Argos, Murcia, etc. et, d'autre part, de l'espèce épigée récente $P$. meridianus abondante au Nord des Pyrénées. Ainsi, tout porte à croire que $P$. beticus n. sp. représente une série de populations rési- duelles (la population de la station 84/7-29, Cueva del Agua, est quelque peu différente de celles de Castellón et Valencia), d'une ancienne espèce oculée de la péninsule ibérique ayant donné de nombreuses espèces souterraines endémiques dans l'aire bétique, mais ayant sans doute également donné naissance à l'espèce récente $P$. meridianus. Il serait intéressant de savoir si $P$. beticus n. sp. subsiste encore dans des localités épigées de cette région bétique.

Proasellus ortizi n. sp.

(Figs. 9-16, 35)

Cette espèce provient de sources, captages et grottes des provinces de Burgos et Santander. Elle est dédiée au Prof. Eugenio Ortiz.

Localités et matériel. - Numéro de collection pour toutes ces stations: ZMA Isop. 105.520.

Prov. Burgos:

Station 83/10-49 (25/10/1983): source captée à Trueba, Espinosa de los Monteros, $34 \mathrm{~km} \mathrm{S.E.} \mathrm{Santander,} \mathrm{alt.} 1100 \mathrm{~m}$, temp. $7,9^{\circ} \mathrm{C}$. Vingt-trois très jeunes Proasellus de 1,1 à $1,8 \mathrm{~mm}$, presque totalement dépigmentés (teinte jaunâtre), rudiments oculaires représentés par deux taches colorées contigües très constantes de chaque côté du céphalon.

Station 83/10-50 (25/10/1983): Fuente Cornejo, à $50 \mathrm{~m} \mathrm{O}$. de la précédente, Trueba, Espinosa de los Monteros, $34 \mathrm{~km} \mathrm{S.E.}$ Santander, alt. $1100 \mathrm{~m}$, temp. $6,9^{\circ} \mathrm{C}$. Vingt-huit jeunes Proasellus de 1,1 à $2,9 \mathrm{~mm}$, les plus grands montrant encore un réseau pigmentaire tergal très ténu. Yeux formés de deux taches pigmentaires comme en station 83/10-49. Le plus grand est un mâle juvénile avec des pléopodes copulateurs montrant qu'il appartient vraisemblablement à la nouvelle espèce.

Station 84/4-11 (07/04/1984): sondage Bou-Rouch, source de Quintanilla de Valdebodres, Merindad de Sotoscueva, $46 \mathrm{~km}$ S.S.E. Santander, alt. $700 \mathrm{~m}$, temp. $9,7^{\circ} \mathrm{C}$. Individus légèrement pigmentés de beige, en particulier dans la région médiale du péréion, yeux bruns, non dissociés: un mâle de $8 \mathrm{~mm}$, une femelle de $5 \mathrm{~mm}$ et quelques jeunes de $2 \mathrm{~mm}$.

Station 84/4-16 (09/04/1984): exsurgence pérenne, Cueva del Agua, Nacimiento del Río Jerea, Quincoces de Yuso, Junta de Oteo, $66 \mathrm{~km} \mathrm{S.E}$. Santander, alt. $700 \mathrm{~m}$, temp. $9,2^{\circ} \mathrm{C}$. Individus presque totalement dépigmentés, yeux bruns réduits: un mâle de $5,3 \mathrm{~mm}$, une femelle de $4,7 \mathrm{~mm}$ et huit jeunes de 1,2 à $2,4 \mathrm{~mm}$.

Station $84 / 4-34=84 / 7-13(24 / 04 / 1984$ et $15 / 07 / 1984)$ : rivière souterraine et émergence temporaires, Cueva la Torcona, Hornillayuso, Merindad de Sotoscueva, $50 \mathrm{~km}$ S.S.E. Santander, alt. $700 \mathrm{~m}$, temp. $10,7^{\circ} \mathrm{C}$. Lot comportant 72 mâles de 3,8 à $11 \mathrm{~mm}$ (moyenne $6,33 \mathrm{~mm}$ ), 78 femelles de 3,2 à $7 \mathrm{~mm}$ (moyenne 5,12 mm; parmi elles, 12 sont des nullipares de 3,2 à 
$3,8 \mathrm{~mm}, 60$ des adultes au repos génital de 4 à $7 \mathrm{~mm}$ et 6 des femelles à marsupium de 4 à $7 \mathrm{~mm}$; la poche incubatrice contient jusqu'à une quinzaine d'oeufs), enfin 3 jeunes de 0,9 à $2,4 \mathrm{~mm}$. Tous les individus portent des rudiments oculaires, en général formés de deux taches pigmentaires. La pigmentation tégumentaire va du grisâtre au blanchâtre, elle n'est jamais totalement absente.

Station 84/7-12 (13/07/1984): rivière souterraine, Cueva Cubio de Berelucho, Las Machorras, Espinosa de Los Monteros, $44 \mathrm{~km}$ S.E. Santander, alt. $900 \mathrm{~m}$, temp. $9,8^{\circ} \mathrm{C}$. Individus pratiquement dépigmentés avec rudiments oculaires à deux masses pigmentaires dissociées: une femelle de $4,1 \mathrm{~mm}$ et cinq jeunes de 1,2 à 2,2 mm (présence d'un jeune Stenasellus virei buchneri (Stammer, 1936) dans le lot).

Station 84/7-?? (14/07/1984): Cueva de Ojo Guarena, Merindad de Sotoscueva, $48 \mathrm{~km}$ S.S.E. Santander. Individus dont les yeux comportent une masse pigmentaire compacte, surmontée de 4-5 corps cristallins de taille réduite; tracé du nerf optique jalonné de granules pigmentaires. Ces yeux pourraient être encore physiologiquement fonctionnels. Corps jaunâtre à blanc presque pur: mâles de 6,$9 ; 6,6 ; 5,7$ et $4,3 \mathrm{~mm}$; femelles de 4,8 ; 4,1 et $4 \mathrm{~mm}$.

Prov. Santander:

Station 83/10-9 (12/10/1983): source du Río Gándara, La Gándara, Soba, $34 \mathrm{~km} \mathrm{S.E.} \mathrm{Santander,} \mathrm{alt.} 580 \mathrm{~m}$, temp. $8,4^{\circ} \mathrm{C}$. Individus pratiquement dépigmentés, yeux ponctiformes, bruns, réduits à leurs cellules pigmentaires: un mâle juvénile de $2,4 \mathrm{~mm}$ et cinq jeunes de 0,9 à $1,5 \mathrm{~mm}$.

Station 83/11-4 (05/11/1983): Cueva de la Cuevona, Jumijar, Quijas, Reocín, $28 \mathrm{~km}$ O.S.O. Santander, alt. $73 \mathrm{~m}$, temp. $12,3^{\circ} \mathrm{C}$. Individus quasi dépigmentés, mais yeux à deux taches pigmentaires séparées, surtout chez les petits: un mâle de $2,6 \mathrm{~mm}$ et cinq femelles de 4,$4 ; 4 ; 3,9 ; 3,5$ et $3,4 \mathrm{~mm}$ (plus un fragment de Stenasellus virei buchneri).

Station 84/2-56 (26/02/1984): perte de Jivero 2, Ozana, Matienzo, $25 \mathrm{~km}$ S.O. Santander, alt. $265 \mathrm{~m}$, temp. $10,0^{\circ} \mathrm{C}$. Quatre mâles de 8,$5 ; 6,9 ; 5,5$ et $5,2 \mathrm{~mm}$ et deux femelles de 4,7 et 4,5 $\mathrm{mm}$. Individus jaunâtres à beige-clair, le réseau de pigment tégumentaire étant très ténu; yeux petits à pigmentation irrégulière, sans cornéules saillantes. Ces Asellides avaient déjà été observés (Ortiz, 1968), mais considérés alors comme un variant de Proasellus coxalis.

Station 84/2-57 (26/02/1984): perte, Cueva del Orillón, Ozana, Matienzo, station-type, très voisine de la précédente, alt. $275 \mathrm{~m}$, temp. $9,6^{\circ} \mathrm{C}$. Lot important d'adultes légèrement pigmentés et tous oculés: 41 mâles de 3 à 7,6 mm (moyenne 4,98); 23 femelles impubères et adultes de 3 à $5,1 \mathrm{~mm}$ (moyenne 3,76); 10 femelles à marsupium vide ou occupé par des oeufs (une quinzaine au maximum) ou embryons, de 2,7 à $4 \mathrm{~mm}$ (moyenne 3,73 ), plus 13 jeunes de 1,3 à $3,3 \mathrm{~mm}$ et des fragments de 5 individus. Holotype: un mâle de $6,9 \mathrm{~mm}$ environ; allotype: une femelle ovigère de $4,2 \mathrm{~mm}$ environ.

Description (individus de la station-type). - Aspect général: Corps trapu, robuste et à chétotaxie bien développée (Fig. 9), assez semblable aux $P$. coxalis de stations obscuricoles. Pigmentation tégumentaire présente mais variable. Les individus de Matienzo sont beige à brun-clair, pigmentation plus marquée sur le céphalon et la base des antennules (Fig. 10) que sur les tergites thoraciques, où elle se cantonne dans la région médiane, au dessus de l'intestin; pléotelson également très peu coloré. Les individus du haut-bassin de l'Ebre sont plus clairs, souvent jaunâtres. Yeux petits, avec plages pigmentaires parfois fragmentées, mais toujours présentes; les corps cristallins sont très réduits. Céphalon plus large que long, avec marge antérieure concave et lobes postmandibulaires nettement saillants, péréion légèrement élargi dans sa zone médiane, plus large que le céphalon et le pléon. Pléotelson subcordiforme à pointe caudale peu marquée (Fig. 9). Coxopodites des péréiopodes largement saillants de part et d'autre des régions pleurales des tergites thoraciques.

Appendices céphaliques: antennules courtes, hampe de 4 et fouet de 9 articles avec une lame olfactive sur les 3 distaux. Antennes relativement longues; hampe de 6 et fouet de 65 articles ou plus. Mandibules typiques du genre avec palpe triarticulé long et normalement sétigère. Maxillules normales: lobe interne avec 5 fortes tiges ciliées distales; lobe externe avec 13 épines denticulées distales et une petite soie subterminale. Maxilles trilobées normales: lobes externe et médian à marge distale portant une rampe de longues lames falciformes unipectinées et lobe interne avec nombreuses soies polymorphes distales.

Maxillipèdes typiques: endite avec nombreux crochets rétinaculaires (6 ou plus); épipodite subquadrangulaire avec des soies lisses sur les marges externe et antérieure; palpe 5-articulé normalement sétigère.

Péréiopodes: péréiopode I faible, à propodite peu renflé et marge palmaire à peine convexe; péréiopode IV raccourci et tordu en "crochet nuptial" comme chez $P$. meridianus ou $P$. coxalis; péréiopodes V, VI et VII de plus en plus longs; dactylopodites II-VII portant jusqu'à 3 épines dactyliennes sternales. Papilles génitales du mâle normalement développées sur le dernier sternite thoracique. 

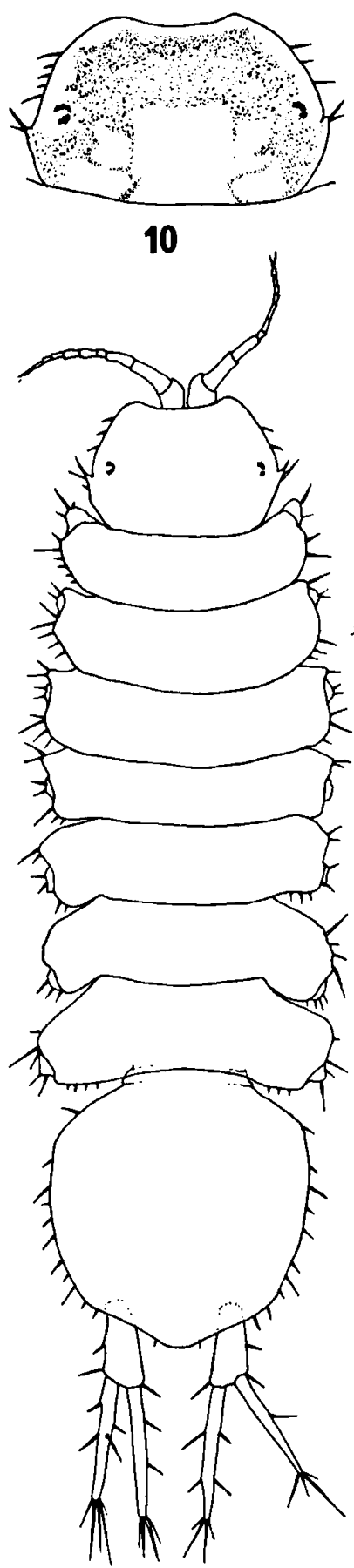

9
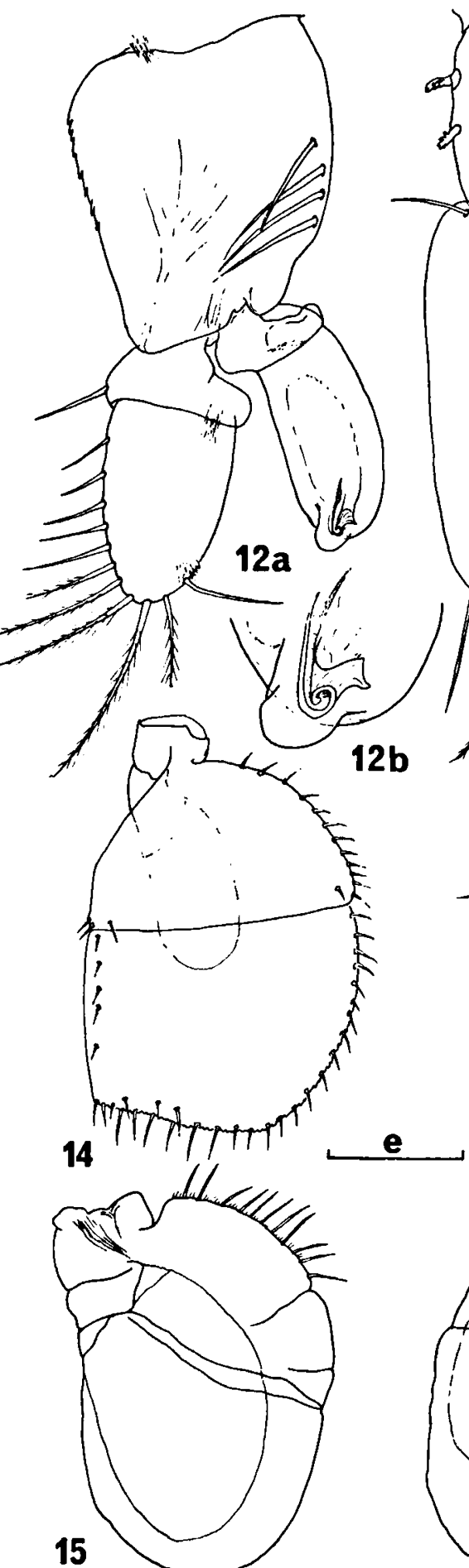

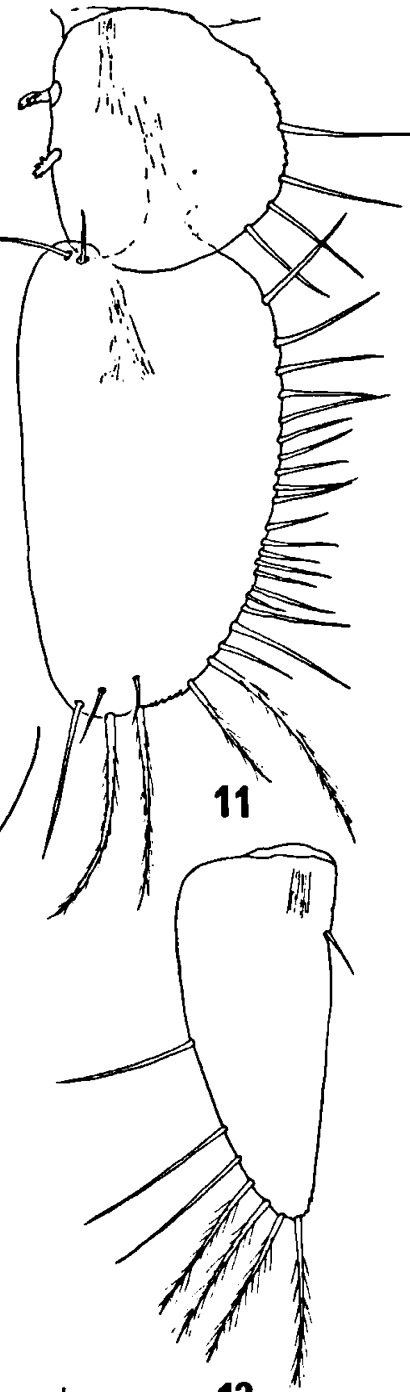

13

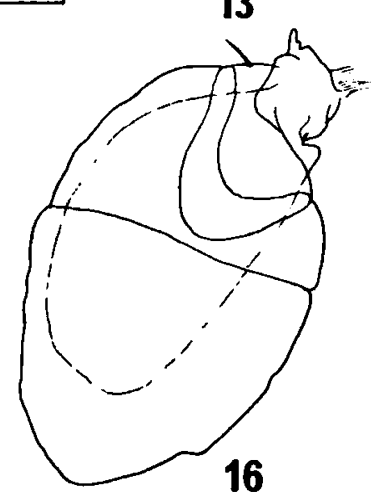

Figs. 9-16. Proasellus ortizi n. sp.: 9, habitus d'un mâle de 7,4' $\mathrm{mm}$ env., e = 1,1 mm; 10, céphalon du même, e = 0,7 mm; 11 , pléopode I du même, $\mathrm{e}=175 \mu \mathrm{m} ; 12 \mathrm{a}$, pléopode II droit, face sternale, du même, $\mathrm{e}=100 \mu \mathrm{m}$; 12b, extrémité de l'endopodite du précédent, e $=50 \mu \mathrm{m} ; 13$, pléopode Il d'une femelle de $5 \mathrm{~mm}$ env., e $=175 \mu \mathrm{m} ; 14$, pléopode III de 9 , e $=465 \mu \mathrm{m} ; 15$, pléopode IV du même, $\mathrm{e}=350 \mu \mathrm{m} ; 16$, pléopode $\mathrm{V}$ du même, $\mathrm{e}=350 \mu \mathrm{m}$. 
Pléopodes I mâles: protopodite aussi long que large avec rétinacle à $1-2$ crochets; marge externe écailleuse et portant jusqu'à 4 longues soies (Fig. 11); rame moyennement allongée, avec une série continue de soies sur les marges externe et distale (seules les plus distales sont longues et plumeuses).

Pléopodes II mâles: protopodite allongé, surtout chez les plus grands individus, avec 4 fortes soies plumeuses submarginales internes; exopodite avec article proximal cupuliforme normal, mais article distal très volumineux, dépassant toujours l'endopodite, avec $10-11$ soies sur les marges externe et distale et une aire pilifère très développée sur la marge interne (Fig. 12a). Endopodite très caractéristique (Fig. 12a), avec une apophyse tergale large et courte, mais peu épaisse, régulièrement arrondie, hyaline, et un goulot portant l'orifice spermatique terminal efférent court et petit, bien en retrait sur l'apophyse, les directions de ces deux éléments ne faisant qu'un angle réduit entre elles (Fig. 12b). Cette architecture, bien différente de celles existant chez les autres espèces oculées $(P$. beticus n. sp., $P$. coiffaiti, $P$. coxalis, $P$. meridianus), justifie à elle seule la création de cette entité spécifique indépendante.

Pléopodes II femelles: subtriangulaires, allongés, à pointe émoussée, avec 1 soie lisse rétinaculaire sur la marge interne (Fig. 13) et environ 7-8 soies plumeuses sur la marge externe et distale.

Pléopodes III: exopodite (opercule) avec marge externe et distale garnies de soies, les distales étant seules légèrement plumeuses. La marge distale, très rectiligne, porte de fines crénelures caractéristiques entre les bases des soies marginales (Fig. 14). Endopodite subovalaire charnu et respiratoire.

Pléopodes IV: exopodite ovalaire, grand, marge externe de son article proximal avec $6-11$ soies et de fines sétules intercalées. Linea conjungens incomplètement visible; linea transversalis bien nette et linea areæ à peine courbe et très proche de la précédente, ce qui délimite une vaste aire charnue interne et distale (Fig. 15). Endopodite ovalaire, plus court que son exopodite, charnu et respiratoire.

Pléopodes V: exopodite ovalaire dont la marge proximale-externe porte jusqu'à 5 soies lisses; linea duplex très basale et linea articularis parfaitement transversale, délimitant une vaste aire distale (Fig. 16). Endopodite subovalaire charnu et respiratoire.

Les portions charnues des pléopodes III, IV et V, surtout les endopodites, peuvent porter des Acinétiens sessiles de type Stylocometes ou pédonculés de type Choanophrya.

Uropodes: un peu moins longs que le pléotelson (Fig. 9).

Affinités. - Par l'architecture de ses pléopodes, $P$. ortizi $\mathrm{n}$. sp. se distingue bien des autres espèces oculées de la région. Il ne montre pas de parenté immédiate avec $P$. coxalis ou $P$. meridianus. En revanche, cette parenté est manifeste avec les espèces anophtalmes et dépigmentées déjà connues dans la région: $P$. cantabricus Henry \& Magniez, 1968, en particulier et peut-être également avec l'espèce obscuricole $P$. variegatus Afonso, $1982 \mathrm{du}$ sud du Portugal. Ainsi, il faut interpréter ces populations obscuricoles que nous avons réunies sous le nom de $P$. ortizi n. sp. comme des peuplements résiduels d'une ancienne espèce oculée et pigmentée ayant peuplé le haut bassin hydrographique de l'Ebre et sans doute les bassins avoisinants.

Il serait souhaitable d'établir le caryotype de $\boldsymbol{P}$. ortizi pour savoir s'il est semblable à celui des espèces anciennes $(2 \mathrm{n}=24$ ou $2 \mathrm{n}=22$, comme c'est le cas pour $P$. racovitzai Henry \& Magniez, 1972), alors que les espèces récentes montrent un nombre plus réduit $(2 \mathrm{n}=12$ pour $P$. coxalis ou $2 \mathrm{n}=10$ pour $P$. meridianus; cf. Henry \& Magniez, 1972b). Sur les plans biologique et physiologique, il serait également souhaitable de vérifier si les différentes populations sont encore interfertiles et si elles sont encore capables de vivre en milieu éclairé, ou même si des populations subsistent dans certaines eaux de surface.

\section{Proasellus ebrensis n. sp.}

(Figs. 17-23, 36)

Le nom spécifique est lié à la situation dans la haute vallée de l'Ebre.

Localité et matériel. - Numéro de collection: ZMA Isop. 105.518 . 
Station 84/4-2 (06/04/1984): sondage Bou-Rouch dans Fuente de Marfil, Valdenoceda, Merindad de Valdevielso, Prov. Burgos, env. $74 \mathrm{~km} \mathrm{S.S.E.} \mathrm{Santander,} \mathrm{alt.} 700 \mathrm{~m}$, temp. $10,8^{\circ} \mathrm{C}$. Lot comprenant des individus dont la pigmentation oculaire est encore relativement bien visible: 4 mâles de 4,$4 ; 4,1 ; 3,8$ (holotype); $3 \mathrm{~mm} ; 2$ femelles ovigères de $3,9 \mathrm{~mm}$ ( 9 pulli) et $3 \mathrm{~mm}$ (env. 15 oeufs), 2 femelles à marsupium vide de 4,9 (allotype) et $3,5 \mathrm{~mm}$ et 2 femelles de $4 \mathrm{~mm}$ et par ailleurs des individus dont les résidus oculaires sont infimes: 15 mâles de 3,0 à $4,1 \mathrm{~mm} ; 7$ femelles de 3,0 à $4,4 \mathrm{~mm}$, certaines avec de petits oostégites; 4 femelles à marsupium de 3,$1 ; 3,3 ; 3,9$ et $4,3 \mathrm{~mm}$; la poche incubatrice pouvant contenir jusqu'à une quinzaine d'embryons; enfin 23 juvéniles de 0,9 à $2,7 \mathrm{~mm}$ et quelques individus détériorés.

Description. - Aspect général: corps blancjaunâtre, pratiquement dépigmenté. Céphalon plus large que long avec marge antérieure légèrement concave et lobes postmandibulaires bien marqués. Quelques individus portent deux yeux ponctiformes bruns, l'organe n'est parfois bien apparent qu'unilatéralement (Fig. 17), mais le plus souvent il n'en subsiste que des taches pigmentaires très ténues, seulement visibles à fort grossissement. L'appareil dioptrique est totalement régressé (cf. De Lattin, 1939; Kosswig \& Kosswig, 1940) et on peut penser que ces organes ne sont plus fonctionnels. Péréion à peine élargi dans sa zone médiane et coxopodites débordant légèrement la marge des tergites. Pléotelson subcordiforme, plus long que large, avec pointe caudale très atténuée.

Appendices céphaliques: antennules courtes, avec hampe de 4 et fouet de 6 articles dont les 3 distaux portent une lame olfactive; antennes avec hampe de 6 articles et fouet en comportant jusqu'à 55 sur les plus grands mâles. Mandibules typiques avec palpe triarticulé bien développé. Maxillules avec lobe externe portant une douzaine de fortes épines dentelées distales plus une soie fine submarginale et lobe interne portant les 5 tiges plumeuses typiques chez les Proasellus. Maxilles trilobées typiques du genre.

Maxillipèdes typiques: endite avec 7 crochets rétinaculaires; épipodite subtrapézoïdal à marge caudale glabre et marge externe portant une vingtaine de soies lisses, plus serrées à l'angle antéroexterne. Palpe à 5 articles normalement sétigères.

Péréiopodes: première paire faible et son propodite peu renflé, avec marge palmaire à peine con- vexe et faiblement armée; péréiopode IV très peu raccourci et peu spécialisé en "crochet nuptial"; dactylopodites des péréiopodes II-VII portant 2 épines dactyliennes sternales. Papilles génitales normales sur le dernier sternite thoracique mâle.

Pléopodes I mâles avec protopodite quadrangulaire un peu plus long que large; marge interne rectiligne avec rétinacle à $1-2$ crochets. Rame régulièrement ovalaire, moyennement allongée (Fig. 18), avec une armature marginale distale et externe d'environ 25 soies toutes courtes.

Pléopodes II mâles avec protopodite subquadrangulaire légèrement plus long que large; les deux rames de longueur équivalente (Fig. 19a). Endopodite (organe copulateur) caractéristique avec une apophyse tergale très massive (Fig. 19b) et dirigée en direction externe, son axe étant pratiquement orthogonal à celui de l'organe copulateur. Goulot court et plutôt grêle, situé nettement en retrait par rapport à l'apophyse (Fig. 19c). L'originalité de l'association de ces structures permet de caractériser une espèce indépendante.

Pléopodes II femelles subtriangulaires à extrémité arrondie, avec quelques soies marginales peu allongées (Fig. 20).

Pléopodes III: exopodite (opercule) avec suture interarticulaire légèrement oblique; marge externe et distale armée de soies lisses, cette marge est non crénelée entre les bases des soies; endopodite charnu et respiratoire petit et étroit (Fig. 21).

Pléopodes IV: exopodite ovalaire à marge externe du premier article régulièrement convexe avec quelques 4 soies lisses et des sétules intermédiaires (Fig. 22); linea conjungens non observable; linea transversalis et linea areæ peu visibles mais très proches, ce qui ménage une très vaste area charnue occupant plus de la moitié de la rame; endopodite oblong, charnu et respiratoire, plus court et plus étroit que le précédent.

Pléopodes V: exopodite ovalaire (Fig. 23), linea duplex très concave, mais peu visible et linea articularis à peine oblique, délimitant une aire distale charnue occupant plus de la moitié de la surface de l'exopodite; endopodite charnu et respiratoire, aussi allongé mais plus étroit que le précédent.

Uropodes: plus courts que le pléotelson; endopodite dépassant légèrement l'exopodite (Fig. 17). 

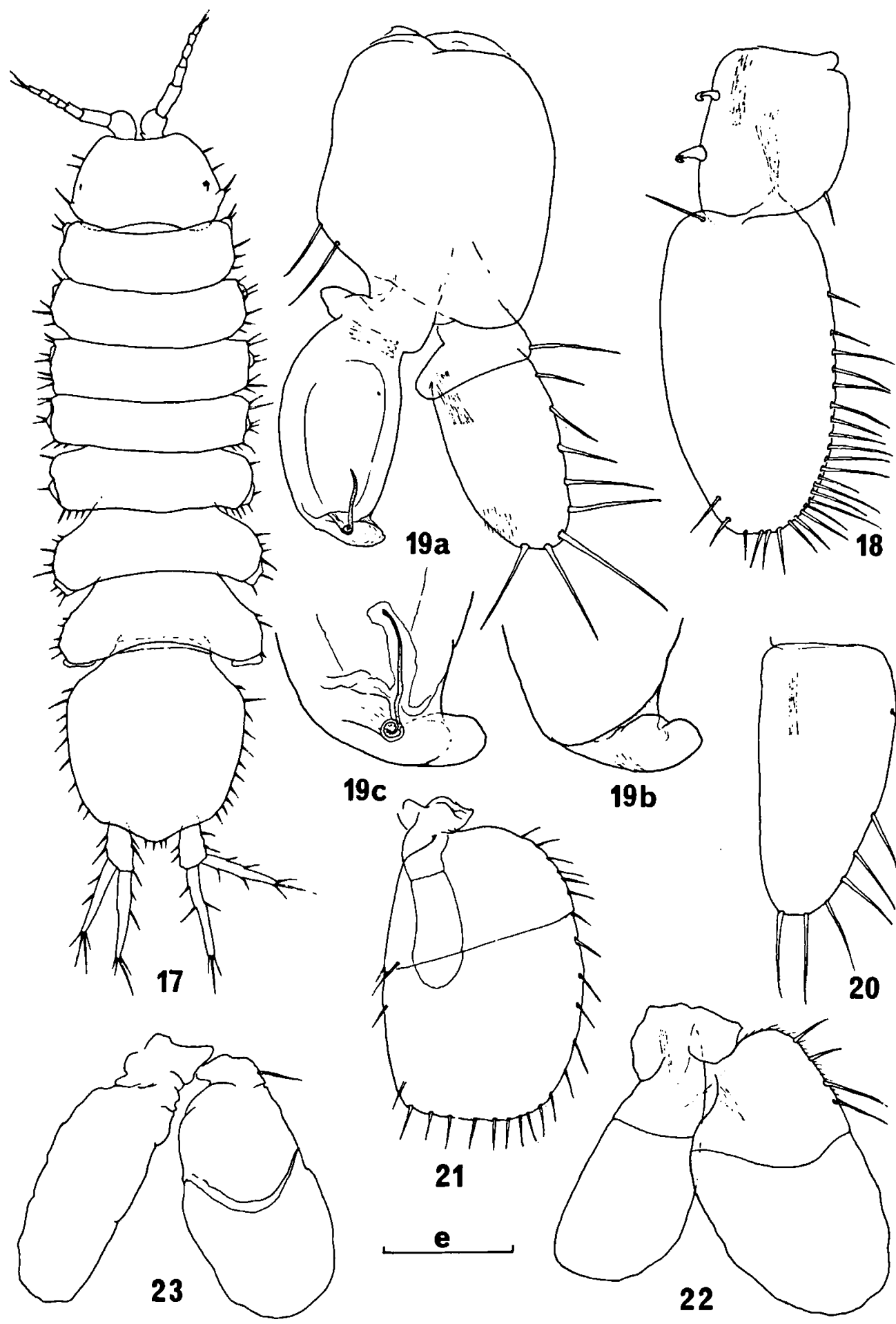
Affinités. $-P$. ebrensis n. sp. appartient au même groupe d'espèces que $P$. ortizi et les espèces stygobies dérivées de la région pyrénéo-cantabre. C'est un variant local de l'espèce-souche oculée qui a acquis une structure reproductrice originale, donc probablement son indépendance génétique, et qui se trouve au seuil de l'anophtalmie totale.

Proasellus aragonensis n. sp.

(Figs. 24-30, 37)

Le nom spécifique est lié à la situation dans la vallée du Río Aragón.

Localités et matériel. - Numéro de collection pour ces deux stations: ZMA Isop. 105.514.

Station 84/6-17 (12/06/1984): puits O. de Villareal de la Canal, $68 \mathrm{~km} \mathrm{~N}$.O. Huesca, alt. $580 \mathrm{~m}$, temp. $13,0^{\circ} \mathrm{C}$. Trois mâles de 6,$5 ; 4,1$ et $3 \mathrm{~mm}$; une femelle adulte de $5,6 \mathrm{~mm}$ et dixneuf jeunes de 1,1 à $2,4 \mathrm{~mm}$.

Station 84/6-18 (13/06/1984): station-type, petite source, E. de la route $C 137$ Burgui-Salvatierra de Esca, rive O. du Río Esca, à $0,2 \mathrm{~km} \mathrm{~N}$. de la limite prov. Zaragoza-Navarra, $52 \mathrm{~km}$ E.S.E. Pamplona, Prov. Navarra, et $13 \mathrm{~km}$ N.O. environ de la station $84 / 6-17$, alt. $610 \mathrm{~m}$, temp. $10,0^{\circ} \mathrm{C}$. Quinze mâles de 6,5 à $3,3 \mathrm{~mm}$ (moyenne: $4,38 \mathrm{~mm}$ ) plus dix-sept femelles de 4,5 à 3,3 $\mathrm{mm}$ (moyenne: $3,78 \mathrm{~mm}$ ) et environ cent soixante-cinq individus (juvéniles, mâles et femelles impubères de 1,1 à $3,2 \mathrm{~mm}$ et quelques adultes incomplets ou déformés). Holotype: un mâle de 6,5 et allotype une femelle à petits oostégites de $4,3 \mathrm{~mm}$ environ.

Description (individus de la station-type). - Aspect général: Asellides très faiblement pigmentés (jaunâtres) et parfois totalement blancs. Yeux rudimentaires à partie dioptrique nulle. Pigmentation oculaire de teinte acajou due à des granules colorés dissociés formant deux petites plages plus ou moins séparées, parfois inégalement développées à droite et à gauche. Corps relativement étroit et allongé avec coxopodites II-VII débordant légèrement les parties pleurales des tergites; pléotelson nettement plus long que large (Fig. 24). Chétotaxie abondante, avec nombreuses soies lisses marginales sur céphalon, péréion et pléotelson.

Appendices céphaliques: antennules courtes: hampe de 4 et fouet de 5 articles, les 3 distaux avec une lame olfactive. Antennes: hampe de 6 et fouet de 45 articles environ. Mandibules typiques avec palpe triarticulé normalement développé et séti- gère. Maxillules: lobe externe avec une douzaine d'épines distales, lobe interne avec les 5 tiges plumeuses typiques. Maxilles trilobées normales avec des lames falciformes monopectinées sur la marge distale des lobes externe et médian.

Maxillipèdes avec endite présentant 5 crochets rétinaculaires; épipodite à surface glabre, portant seulement une quinzaine de courtes soies lisses sur les marges antérieure et externe. Palpe pentaarticulé normalement sétigère.

Péréiopodes: péréiopode I grêle, son propodite à peine renflé, avec marge palmaire faiblement armée; péréiopode IV à peine raccourci et à conformation en "crochet nuptial" à peine ébauchée; péréiopodes II-VII avec 2 épines dactyliennes sternales.

Pléopodes I mâles: protopodite aussi long que large, marges interne et externe régulièrement convexes, rétinacle formé d'un crochet. Exopodite ovalaire avec en général une seule soie longue et plumeuse sur la marge distale, les autres étant courtes et lisses (Fig. 25).

Pléopodes II mâles: protopodite régulièrement quadrangulaire, aussi long que large, avec 4 tiges submarginales internes (Fig. 26a). Exopodite biarticulé, de longueur similaire à celle de l'autre rame, avec quelques soies marginales. Endopodite (Fig. 37) bananiforme à marge interne convexe. Apophyse tergale massive, très large à sa base, à marge distale régulièrement convexe. Goulot (Fig. 26b) de petit diamètre, court, inséré en retrait par rapport à l'apophyse et orifice spermatique efférent distal petit et à peine marginé.

Pléopodes II femelles libres, subtriangulaires, allongés, avec une demi-douzaine de longues soies plumeuses sur la marge distale-externe (Fig. 27).

Pléopodes III: exopodite opercule (Fig. 28), à marge distale subrectiligne crénelée comme chez $P$. ortizi n. sp.; endopodite petit, charnu et respiratoire.

Pléopodes IV: endopodite ovalaire (Fig. 29), marge proximale-externe avec des sétules et quelques soies lisses. Linea conjungens partiellement visible, linea transversalis et linea areæ très proches l'une de l'autre et très proximales, ménageant une très vaste area charnue distale. Endopodite ovalaire, petit et respiratoire. 

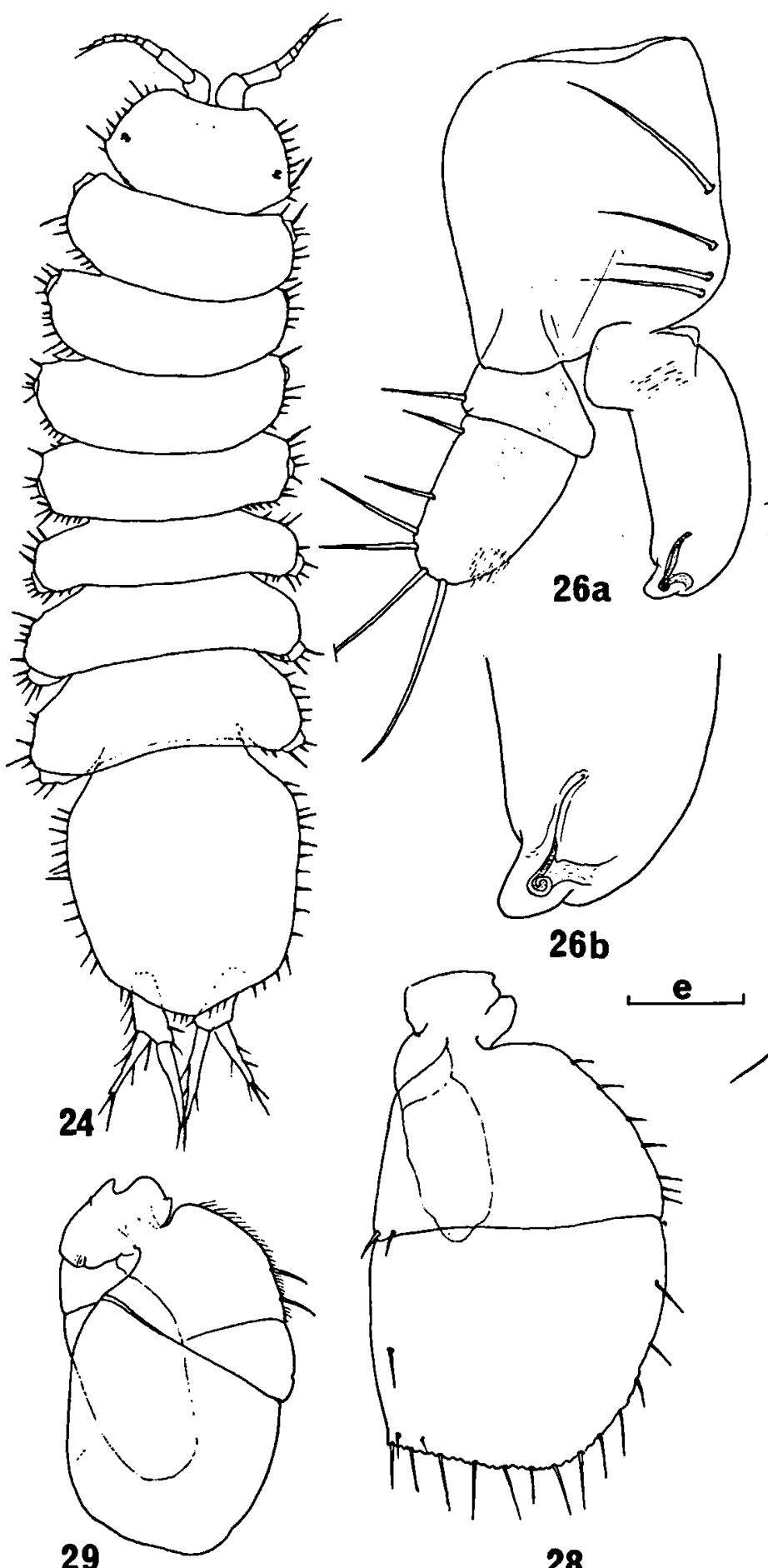

28

30

Figs. 24-30. Proasellus aragonensis n. sp.: 24, habitus d'un mâle de 4,3 mm env., e = 0,55 mm; 25 , pléopode I du même, e $=100$ $\mu \mathrm{m} ; 26 \mathrm{a}$, pléopode II gauche, face sternale, du même, e = $75 \mu \mathrm{m} ; 26 \mathrm{~b}$, extrémité de l'endopodite du même, e = 35 $\mu \mathrm{m} ; 27$, pléopode II d'une femelle de $4,4 \mathrm{~mm}$ env., $e=150 \mu \mathrm{m} ; 28,29,30$, pléopodes III, IV et V de $24, e=220 \mu \mathrm{m}$. 


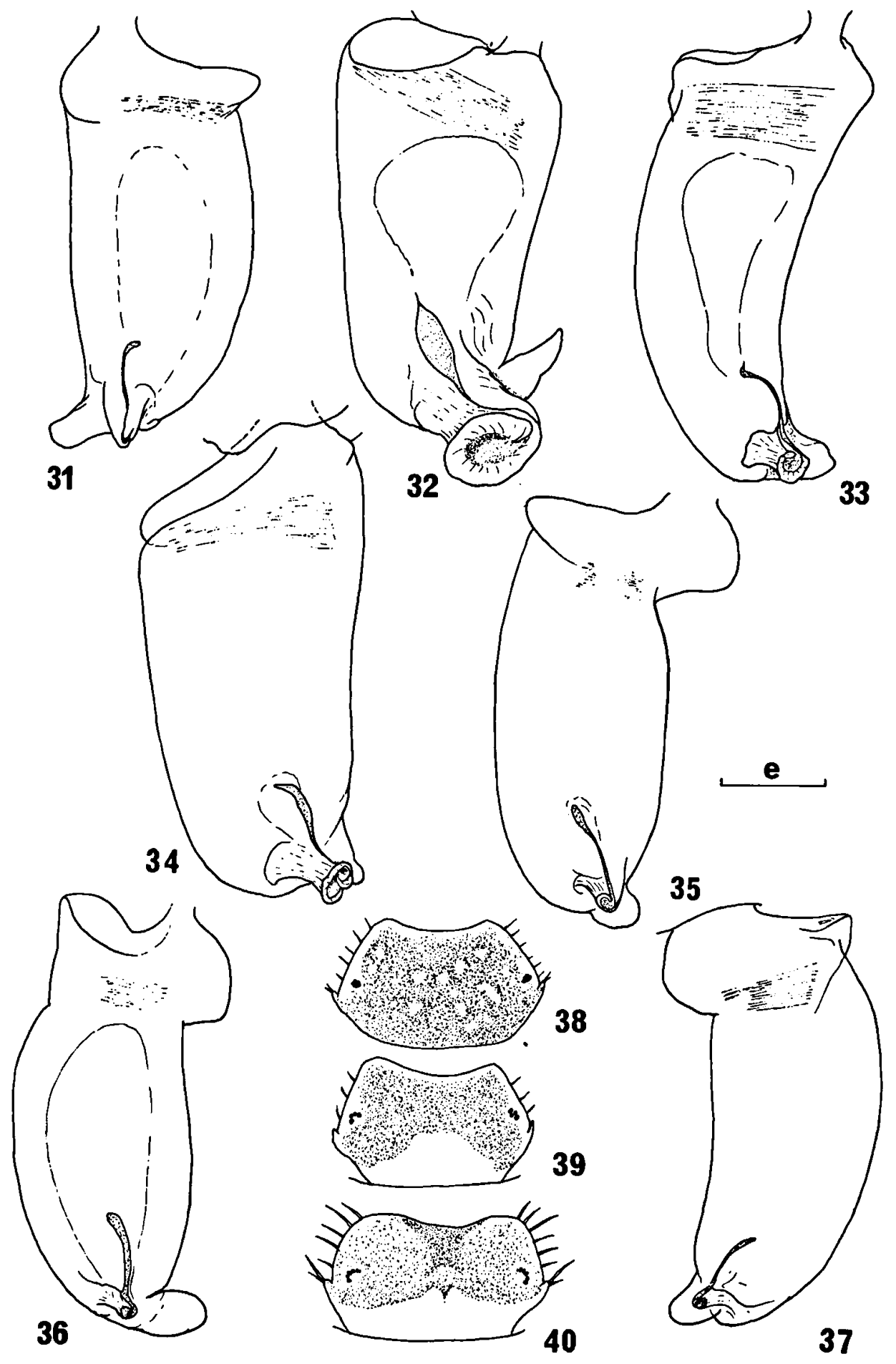

Figs. 31-40. Comparaison de l'endopodite du pléopode II mâle des espèces citées: $31, P$. coxalis, e $=50 \mu \mathrm{m} ; 32, P$. meridianus, e $=$ $100 \mu \mathrm{m} ; 33, P$. coiffaiti, e $=100 \mu \mathrm{m} ; 34, P$. beticus, $\mathrm{e}=60 \mu \mathrm{m} ; 35, P$. ortizi, e $=50 \mu \mathrm{m} ; 36, P$. ebrensis, $\mathrm{e}=55 \mu \mathrm{m} ; 37$, . aragonensis, $e=45 \mu \mathrm{m}$.

Comparaison de la pigmentation céphalique: $38, P$. coxalis; $39, P$. meridianus; $40, P$. coiffaiti. 


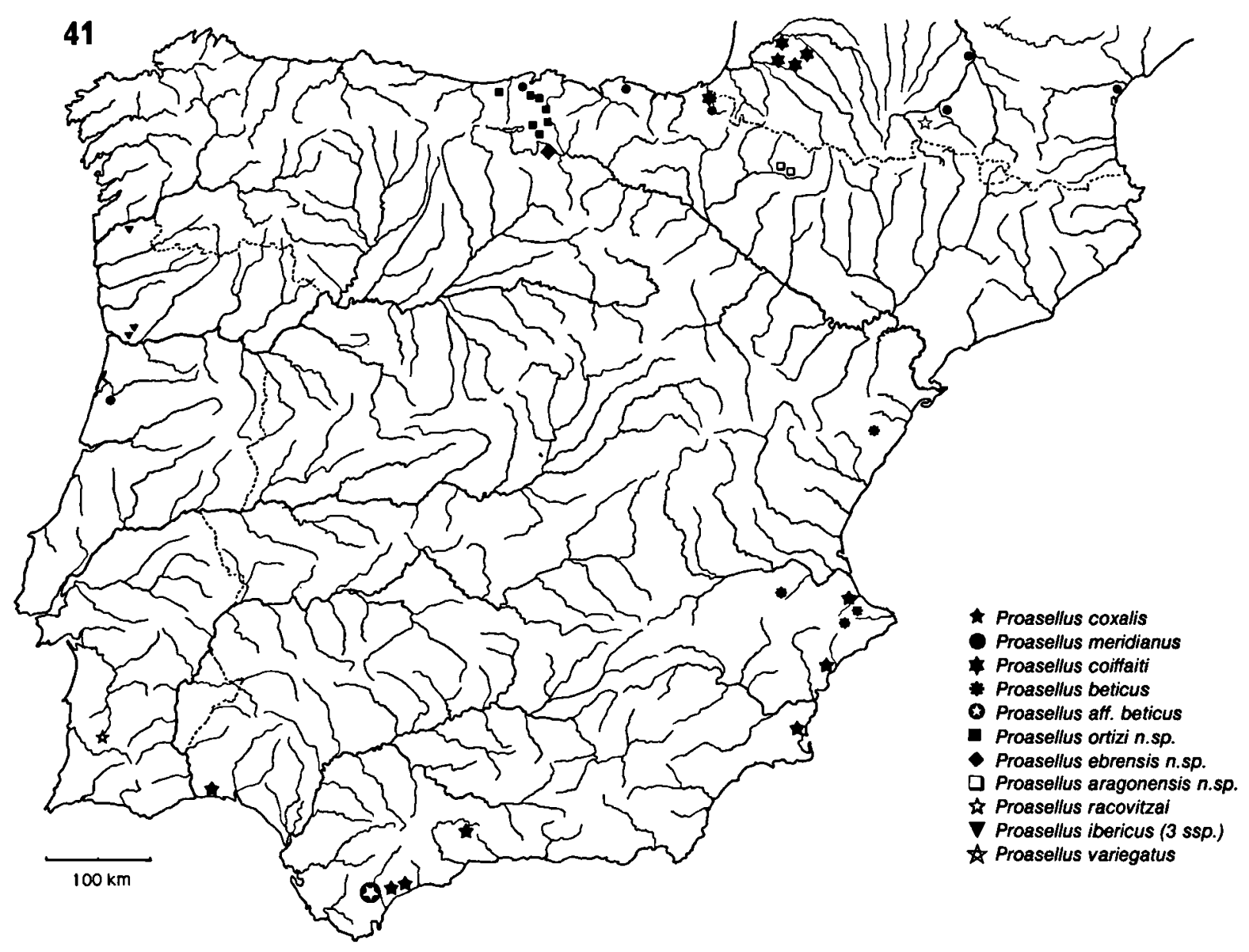

Fig. 41. Carte hydrographique de la péninsule ibérique et répartition des Proasellus cités.

Pléopodes V: exopodite régulièrement ovalaire, linea duplex peu visible, mais très concave et très proximale; linea articularis parfaitement transverse et proximale, ce qui délimite un vaste article distal charnu (Fig. 30).

Uropodes: beaucoup plus courts que le pléotelson (Fig. 24).

Affinités. - $\boldsymbol{P}$. aragonensis $\mathrm{n}$. sp. apparaît nettement apparenté à $P$. ortizi décrit plus haut, mais son adaptation à la vie souterraine est plus poussée et l'extrémité de son pléopode copulateur est suffisamment différente pour l'en isoler. Cette architecture l'apparente par ailleurs étroitement aux espèces stygobies connues du versant Nord des Pyrénées et du bassin aquitain: $P$. albigensis (Magniez, 1965), $P$. spelaeus, $P$. vandeli Magniez \& Hen- ri, 1969 et surtout $P$. aquaecalidae (Racovitza, 1922). On sait que cette dernière espèce présente une répartition transpyrénéenne (hauts-bassins du Gave d'Aspe et du Río Aragón à Villanua, cf. Henry \& Magniez, 1983); l'existence du microphtalme $\boldsymbol{P}$. aragonensis pourrait donc bien expliquer l'origine de ces espèces anophtalmes pyrénéoaquitaines.

\section{Note finale}

Afin de faciliter la détermination des Proasellus oculés ou microphtalmes de la péninsule ibérique, des schémas comparatifs de l'endopodite du pléopode 2 du mâle adulte (organe copulateur) sont représentés côte à côte: Fig. $31=P$. coxalis; $32=$ $P$. meridianus; $33=P$. coiffaiti $34=P$. beticus; 
$35=P$. ortizi; $36=P$. ebrensis; $37=P$. aragonensis. L'espèce lusitanienne $P$. ibericus n'est pas figurée.

\section{Conclusions}

La découverte de $P$. beticus n. sp. et de $P$. ortizi n. sp. nous invite à réfléchir au problème biogéographique et évolutif posé par le genre Proasellus. Si l'on fait abstraction des espèces de surface très expansives ( $P$. coxalis et $P$. meridianus), d'origine vraisemblablement récente, les eaux de surface de la péninsule ibérique ont dû être peuplées autrefois par deux grandes espèces pigmentées-oculées, bien différentes et indépendantes l'une de l'autre: appelons-les: "preortizi" et "prebeticus".

La première, réduite à des populations-relictes confinées dans des biotopes-limites ou obscurs (l'espèce $P$. ortizi actuelle) semble aussi avoir donné des variants encore microphtalmes, tels que $\boldsymbol{P}$. ebrensis ou $P$. aragonensis, mais aussi de nombreuses espèces totalement dépigmentées et anophtalmes qui habitent le bassin aquitain, l'ouest de la chaîne pyrénéenne et la Cordillère cantabrique ( $P$. albigensis, $P$. aquaecalidae, $P$. cantabricus, $P$. chappuisi Henry \& Magniez, 1968, $P$. spelaeus, $P$. vandeli ...).

La seconde, dont les relictes oculées constituent $P$. beticus actuel, semble avoir fourni de nombreuses espèces stygobies (telles que $P$. jaloniacus, $P$. solanasi, etc.), endémiques de la moitié S.E. de la péninsule. Mais $P$. beticus présente une autre particularité: elle semble nettement apparentée à tout un réseau d'espèces extra-ibériques encore oculées paraissant toutes très endémiques, souvent inféodées à des biotopes-limites obscurs. Ce sont: $P$. racovitzai Henry \& Magniez, 1972 des Pyrénées centrales; $P$. monsferratus (Braga, 1948) de Kabylie; $\boldsymbol{P}$. istrianus (Stammer, 1932) de l'Istrie; $\boldsymbol{P}$. remyi (Monod, 1932) du Lac d'Ohrid; $P$. gjorgjevici (Karaman, 1933) du lac d'Ohrid; $P$. monodi (Strouhal, 1942) de Grèce; $P$. pribenicensis Flašarova, 1977 de Slovaquie et du nord de la Transylvanie; et $P$. infirmus (Birstein, 1936) de Géorgie.

Ces espèces présentent des traits morphologiques communs, en particulier au niveau des pléopodes I et II mâles: pléopode I à exopodite relativement court et à soies marginales courtes et lisses, endopodite copulateur à apophyse tergale courte et épaisse, goulot court, de diamètre important, marginé et plus ou moins évasé en corolle, ce qui évoque une parenté avec l'espèce récente $P$. meridianus.

Il aurait donc pu exister, dans toute cette aire péri-méditerranéenne, une espèce ancienne, ou un essaim d'espèces de morphologie extrêmement voisine, dont il ne subsiste que des populations très dissociées, et d'où dérivaient un certain nombre d'espèces stygobies, en particulier du S.E. de l'Espagne ou des Balkans. Par la suite, $P$. coxalis est venu s'installer dans tous les territoires périméditerranéens, et donner, à côté de peuplements superficiels nombreux, des populations obscuricoles de phénotypes très divers, ce qui complique l'analyse de la faune asellidienne.

A côté de ces cas, qui correspondent à l'évolution intervenue en Europe méridionale, au ProcheOrient et en Afrique du Nord, le genre Proasellus a dû comporter, en particulier en Europe moyenne, des espèces superficielles aujourd'hui totalement éliminées par les phases glaciaires, et dont les lignées ne se perpétuent plus que par des espèces hypogées: groupe cavaticus, par exemple. Cela permet d'expliquer aussi l'existence dans cette même Europe moyenne, de quelques espèces totalement isolées que l'on a du mal à rattacher à une forme épigée actuelle: cas de $\boldsymbol{P}$. hermallensis (Arcangeli, 1938) du bassin de la Meuse. Ainsi, une meilleure connaissance taxonomique, biologique et écologique des espèces superficielles ou présentant encore une pigmentation partielle et des rudiments oculaires ne peut qu'améliorer la compréhension de l'histoire évolutive des Asellides, et en particulier celle de l'origine des stygobies européens et périméditerranéens.

\section{Références}

Afonso, O., 1982. Contribution à l'étude des Asellides (Crustacea, Isopoda) du Portugal - Description d'une nouvelle espèce. Publicações Inst. Zool. Dr. Augusto Nobre, 172: 1-20.

Braga, J.M., 1946. Quelques Asellides nouveaux du Portugal. Mems. Estud. Mus. zool. Univ. Coimbra, 173: 1-25. 
Braga, J.M., 1956. Un Asellus nouveau de l'étang de Fermentelos (Portugal). Mems. Estud. Mus. zool. Univ. Coimbra, 245: 1-12.

Ermin, R., 1944. Über Farbe und Faerbung bei Asseln. Istanb. Univ. Fen Fak. Mecm., (B) 9 (3): 181-203.

Henry, J.-P., 1967. Un nouvel exemple de colonisation du domaine souterrain par un Aselle épigé. Spelunca, Mém., 5: 262-264.

Henry, J.-P., J.J. Lewis \& G. Magniez, 1986. Isopoda: Asellota: Aselloidea, Gnathostenetroidoidea, Stenetrioidea. In: L. Botosaneanu (ed.), Stygofauna mundi. A faunistic, distributional, and ecological synthesis of the world fauna inhabiting subterranean waters (including the marine interstitial): 434-464 (E.J. Brill/Dr. W. Backhuys, Leiden).

Henry, J.-P. \& G. Magniez, 1972a. Un Aselle cavernicole d'Espagne méridionale: Proasellus solanasi n. sp. (Crustacea, Isopoda, Asellota). Int. J. Speleol., 4: 37-43.

Henry, J.-P. \& G. Magniez, 1972b. Observations sur un Aselle obscuricole de France: Proasellus racovitzai n. sp. (Crustacea, Isopoda, Asellota). Int. J. Speleol., 4: 171-188.

Henry, J.-P. \& G. Magniez, 1972c. Un Aselle endémique pigmenté et oculé de France: Proasellus coiffaiti n. sp. (Crustacea, Isopoda, Asellota). Annls. Spéléol., 27 (1): 195-202.

Henry, J.-P. \& G. Magniez, 1978. Premier Proasellus hyporhéique d'Espagne: $\mathbf{P}$. jaloniacus n. sp., (Crustacea, lsopoda, Asellota). Int. J. Speleol., 9: 125-130.

Henry, J.-P. \& G. Magniez, 1982. Nouveaux Asellides hypogés du Sud-Est de l'Espagne (Crustacea, Isopoda, Asellota). Bull. zoöl. Mus. Univ. Amsterdam, 9 (6): 37-45.

Henry, J.-P. \& G. Magniez, 1983. Introduction pratique à la systématique des organismes des eaux continentales françaises, 4. Crustacés Isopodes (principalement Asellotes). Bull. mens. Soc. linn. Lyon, 52 (10): 319-357.

Henry, J.-P. \& G. Magniez, 1987. Isopodes Aselloïdes stygobies d'Espagne récoltés par J. Notenboom et I. Meijers. I - Le genre Synasellus et description de trois nouvelles espèces. Stygologia, 3 (4): 331-344.

Henry, J.-P. \& G. Magniez, 1988. Isopodes Aselloïdes stygobies d'Espagne récoltés par J. Notenboom et I. Meijers. II - Le genre Bragasellus et description de sept nouvelles espèces. Stygologia, 4 (4): 332-362.

Hurk, P. van den \& R. Leys, 1986. Descriptions of the localities visited during a stygofauna collecting trip to southern Spain
(Andalusia), June-July 1985, with preliminary results of collected taxa: 1-14 (unpublished Report, Institute of Taxonomic Zoology, University of Amsterdam).

Kosswig, C. \& L. Kosswig, 1940. Die Variabilität bei Asellus aquaticus, unter besonderer Berücksichtigung der Variabilität in isolierten unter- und oberirdischen Populationen. Istanb. Univ. Fen Fak. Mecm., (n.S.) 5 (1-2): 78-132.

Lattin, G. de, 1939. Untersuchungen an Isopodenaugen. Zool. Jb., (Anat. Ontog.) 65: 417-468.

Margalef, R., 1950. Algunos Crustáceos interesantes de las aguas dulces y salobres de España. Publnes. Inst. Biol. apl., 7: $131-152$.

Margalef, R., 1953. Los Crustáceos de las aguas continentales ibericas. Biologia de las Aguas continentales, 10: 1-243 (Inst. Forest. Invest. exp. Minist. Agr., Madrid).

Notenboom, J., 1986. Research on the groundwater fauna of Spain: List of stations visited in 1986: 1-6 (unpublished Report, Institute of Taxonomic Zoology, University of Amsterdam).

Notenboom, J. \& 1. Meijers, 1985a. Investigaciones sobre la fauna de las aguas subterráneas de España: lista de estaciones y primeros resultados. Verslagen en technische Gegevens, Instituut voor Taxonomische Zoölogie (Zoölogisch Museum) Universiteit van Amsterdam, 42: 1-93.

Notenboom, J. \& I. Meijers, 1985b. Groundwater stations sampled in Spain during summer 1985: 1-3 (unpublished Report, Institute of Taxonomic Zoology, University of Amsterdam).

Ortiz, E., 1968. Algunos Crustáceos y Miriopodos cavernicolas de la región de Matienzo (Santander). Cuadernos Espeleologia, Santander, 4 (3): 85-87.

Racovitza, E.-G., 1919. Notes sur les Isopodes: 3. Asellus banyulensis n. sp. - 4. A. coxalis Dollfus. - 5. A. coxalis peyerimhoffi n. subsp. Archs. Zool. expér. gén., 58 (Notes Revue) (3): 49-77.

Sabater, F. \& J. De Manuel, 1988. Interstitial Isopoda of the river Ter (Catalonia, N.E. Spain) and description of two new species, Microcharon margalefi n. sp. and Proasellus phreaticus $n$. sp. Stygologia, 4 (3): 267-278.

Reçu: le 11 septembre 1991

Révision: le 24 décembre 1991 